\title{
Prevalence and associated factors of malnutrition among adult hospitalized patients at Amhara National Regional State Referral Hospitals, Ethiopia
}

\author{
Alemtsehay Haile ${ }^{i *}$, Mignote $\mathrm{Hailu}^{2}$ and Eleni Tesfaye ${ }^{2}$ \\ ${ }^{1}$ Gondar University Referral Hospital, Gondar, Ethiopia \\ ${ }^{2}$ Department of Nursing, College of Medicine and Health Sciences University of Gondar, Gondar, Ethiopia
}

\begin{abstract}
Based on numerous reports worldwide, an estimated $13 \%$ to $69 \%$ of hospitalized patients are malnourished. Even though hospitalized patients are more prone to malnutrition; Clinical nutrition and nutritional assessment are often neglected components of the health service practice. To assess prevalence and associated factors of malnutrition among adult hospitalized patients. Institution based cross- sectional study was deployed and stratified random sampling technique was used with a total of 403 patients were included in the analysis. Weight and height of the patient was measured to calculate body mass index and the study subject was interviewed based on the questionnaire. The degree of association was assessed using odds ratio with $95 \%$ confidence interval and $\mathrm{P}$ value. Both bivariate and Multivariate logistic regression were used to identify the associated factors. The prevalence of malnutrition of the study participant was found to be $55.6 \%$. Marital status, Education level, Weight loss, HIV/AIDS co-infection and Individual dietary diversity score were the identified factors affecting under nutrition. Prevalence of malnutrition among adult hospitalized patients is found to be high. Therefore, great emphasis has to be given for nutritional management of adult hospitalized patients. Furthermore additional researches should also be conducted.
\end{abstract}

\section{Background}

Globally, malnutrition is the most important risk factor for illness and death [1]. In 1859, Florence Nightingale wrote about hospitalized soldiers during the Crimea war, starving amongst plenty of food. Over 100 years later, beginning from the 1970s, numerous authors have reported malnutrition rates in hospital patients to be approximately $35 \%$, with 30 to $55 \%$ of patients entering acute hospitals being at risk of malnutrition [2].

World Health Organization (WHO) estimates that by 2015 prevalence of malnutrition worldwide will be $17.6 \%$ - with the vast majority living in developing countries in southern Asia and subSaharan Africa. An additional 29\% will have stunted growth due to poor nutrition $[1,3]$.

The Europeans study assessed 5,051 patients admitted to European hospitals and found that $32.6 \%$ of hospitalized patients at risk of malnutrition [4].

According to United Kingdom the prevalence of hospital malnutrition is difficult. Over the past 34 years, researchers have used different criteria to define it, and studies have been conducted worldwide on populations that differ in socioeconomic status, educational level, age group, and severity of illness; study group size also varies. Based on many reports worldwide, an estimated $13 \%$ to $69 \%$ of hospitalized patients are malnourished [5].

In Singapore study shown that malnourished patients (29\%) had longer hospital stays ( $6.9 \pm 7.3$ days vs. $4.6 \pm 5.6$ days) and was more likely to be readmitted within 15 days. The mean difference between actual cost of hospitalization and the average cost for malnourished patients was greater than well-nourished patients. Mortality was higher in malnourished patients at 1 year (34\% vs. $4.1 \%), 2$ years $(42.6 \%$ vs. $6.7 \%)$ and 3 years ( $48.5 \%$ vs. $9.9 \%)$; for all. Overall, malnutrition was a significant predictor of mortality [6].

In Brazil studies shown that malnutrition occurred in $60.7 \%$ and the variables associated with malnutrition were: recent and involuntary weight loss, apparent bony structure, decreased appetite, diarrhea, inadequate energy intake and male sex [7].

In Spain, studies have shown that prevalence of malnutrition in hospitalized patient's ranges from $30-50 \%$, with increasing rates among patients with longer in-stays [8].

Generally overweight is less prevalent than under nutrition in the developing world, particularly in rural areas, and that it is concentrated in higher socioeconomic status groups [9].

However, this study will be important to show the prevalence of malnutrition on adult hospitalized patient and the possible risk factors that can be used to know nutritional status, to give nutritional advice, therapy and support to the hospitalized patients.

Correspondence to: Alemtsehay Haile, Gondar University Referral Hospital, Gondar, Ethiopia; E-mail: alemtsehay.haile@yahoo.com

Key words: malnutrition, hospitalized patient, body mass index

Received: April 19, 2015; Accepted: May 25, 2015; Published: May 27, 2015 


\section{Methods}

Institution-based cross-sectional study was conducted from March to April, 2014 at 5 referral hospitals found in Amhara regional state. Amhara regional state is one of the 9 regional states of Ethiopia. Its capital city, Bahir Dar, is found $578 \mathrm{~km}$ far from Addis Abeba to the Northwest of Ethiopia. In the region there are a total of 5 referral hospitals (Gondar university referral hospital, Felege Hiwot referral hospital, Debremarkos referral hospital, Debrebrhan referral hospital and Dessie referral hospital). Each hospital serves as a referral center for more than five million people in the catchment area. Four of the hospitals are under the administration of Amhara Regional state health bureau and the other one (Gondar University referral hospital) is a university hospital which is under the administration of Federal Ministry of Education

\section{Study design, participants, and sampling procedure}

Institutional based cross-sectional study design was used and study participants were adult hospitalized patients admitted in Amhara National Regional State Referral Hospitals. The sample size was determined using the formula for single population proportion with a $95 \%$ confidence interval, a precision of $5 \%$, and an assumed prevalence of malnutrition $50 \%(0.5)$ to get a maximum sample size as there was no previous study conducted similar to this study. After adding 5\% non response rate the final sample size was found to be 403 . The study subject was selected proportionally to size allocation to give equal chance to each referral hospitals. Additionally to select patients among different wards, the researchers again used the same method and then stratified random sampling technique was utilized. Data were collected using a pretested interview based questionnaire and clinical measurement of weight and height to determine the body mass index.

\section{Data management and analysis}

Data was entered in to EPI-INFO version 3.5.1 statistical software and then exported to SPSS for windows version 20 for further analysis. Body mass index was used to categorize patients as normal and malnourished. In this study Body Mass

Index $<18.5$ was categorized as malnourished. Bivariate analysis was used primarily to check which variables have association with malnutrition individually. Variables found to have association were then entered into multiple logistic regression models for controlling the possible effect of confounders and finally the variables which have significant association were identified on the basis of OR, with $95 \% \mathrm{CI}$ and $P$ value.

\section{Ethical consideration}

The study obtained ethical approval from the department of nursing ethical review committee. Permission was obtained from Chief Executive Officers (CEO) of each referral Hospitals. Written informed consent was obtained from each study participants. The malnourished hospitalized patients who were not on nutritional support during data collection period were given advice on the appropriate dietary intake and referred for further and better management.

\section{Results}

\section{Response coverage}

A total of 403 adult hospitalized patients were completed and returned the questionnaires, giving a response rate of $100 \%$. The majorities 136 (33.7\%), 123 (30.5\%) of the respondents were from University of Gondar and Felegehiwot Hospitals respectively.

\section{Socio demographic characteristics of respondents}

Among the total of 403 respondents 211(52.4\%) were females. The mean age of the respondents was $39.94(\mathrm{SD} \pm 16.6)$. Majority 333 $(82.6 \%)$ were followers of orthodox Christianity. Two hundred sixty four (65.5\%) were married. A higher proportion of respondents 267 $(66.3 \%)$ were Illiterate. The mean family size of respondents was 4.60 . The wealth index results showed that $131(32.5 \%), 136(33.7 \%)$ and $136(33.7 \%)$ of patients were poor, medium and rich respectively. Majority of patients 213 (52.9\%) were farmers (Table 1).

\section{Prevalence of malnutrition}

Among the total of 403 adult hospitalized patients participate in this Study, 224 (55.6\%) were malnourished and 44.4\% normal (Figure 1).

\section{Factors associating with nutritional status of hospitalized patient}

Adult hospitalized patient with low dietary diversity score were 3.6 times more likely to be malnourished when compared to those with high diversity score [AOR 3.690, 95\%CI (1.946,6.995)]. Respondents who were medium diversity score are 1.8 times more likely to be malnourished when compared to those with high diversity score [AOR 1.816 95\% CI $(1.024,3.220)]$.

Education was significant variable; Illiterate patients were 2.0 times more likely to be malnourished as compared to literates [AOR 2.046, $95 \%$ CI $(1.261,3.321)]$.

Table 1. Socio demographic characteristics of prevalence of malnutrition among adult Hospitalized patients at Amhara National Regional state Referral Hospitals, 2014.

\begin{tabular}{|c|c|c|c|}
\hline \multicolumn{2}{|c|}{ Characteristics } & \multirow{2}{*}{$\begin{array}{c}\text { Frequency } \\
264\end{array}$} & \multirow{2}{*}{$\begin{array}{c}\text { Percent } \\
65.5\end{array}$} \\
\hline & Married & & \\
\hline \multirow[t]{2}{*}{ Marital Status } & Single & 87 & 21.6 \\
\hline & Divorced & 52 & 12.9 \\
\hline \multirow[t]{3}{*}{ Age } & $18-40$ & 181 & 44.9 \\
\hline & $41-64$ & 164 & 40.7 \\
\hline & $>64$ & 58 & 14.4 \\
\hline \multirow[t]{3}{*}{ Religion } & Orthodox & 333 & 82.6 \\
\hline & Muslim & 64 & 15.9 \\
\hline & Protestant & 6 & 1.5 \\
\hline \multirow[t]{4}{*}{ Education } & Illiterate & 267 & 66.3 \\
\hline & Literate & 136 & 33.7 \\
\hline & $<4$ & 140 & 34.7 \\
\hline & $\geq 4$ & 263 & 65.3 \\
\hline \multirow[t]{4}{*}{ Sex } & Male & 192 & 47.6 \\
\hline & Female & 211 & 52.4 \\
\hline & Farmer & 213 & 52.9 \\
\hline & Merchant & 46 & 11.4 \\
\hline \multirow[t]{4}{*}{ Occupation } & $\begin{array}{l}\text { Government } \\
\text { employee }\end{array}$ & 32 & 7.9 \\
\hline & Daily Laborer & 20 & 5.0 \\
\hline & Student & 24 & 6.0 \\
\hline & No Job & 68 & 16.9 \\
\hline \multirow[t]{3}{*}{ Wealth Index } & Poor & 131 & 32.5 \\
\hline & Medium & 136 & 33.7 \\
\hline & Rich & 136 & 33.7 \\
\hline
\end{tabular}




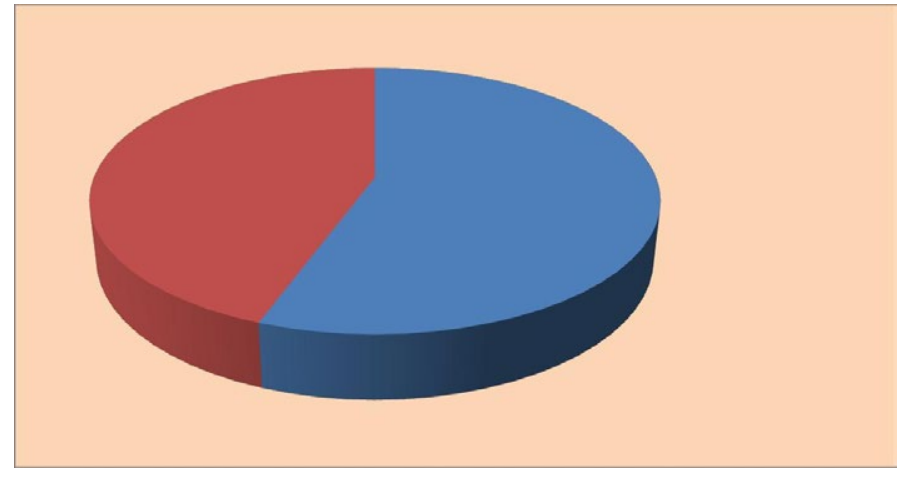

Figure 1. Prevalence of malnutrition among adult hospitalized patients in Amhara National Regional State Referral Hospitals, 2014.

Table 2. Characteristics of respondents with respect to factors associated with malnutrition, 2014

\begin{tabular}{|c|c|c|c|c|c|}
\hline \multirow[b]{2}{*}{ Variables } & & \multicolumn{2}{|c|}{ Normal } & \multicolumn{2}{|c|}{ Malnourished } \\
\hline & & Frequency & Percent & Frequency & percent \\
\hline \multirow[t]{3}{*}{ Marital Status } & Married & 136 & 76.0 & 128 & 57.1 \\
\hline & Single & 29 & 16.2 & 58 & 25.9 \\
\hline & Divorced & 14 & 7.8 & 38 & 17.0 \\
\hline \multirow[t]{3}{*}{ Age } & $18-40$ & 70 & 39.1 & 111 & 49.6 \\
\hline & $41-64$ & 79 & 44.1 & 85 & 37.9 \\
\hline & $>64$ & 30 & 16.8 & 28 & 12.5 \\
\hline \multirow[t]{2}{*}{ Education } & Illiterate & 107 & 59.8 & 160 & 71.4 \\
\hline & Literate & 72 & 40.2 & 64 & 28.6 \\
\hline Individual Dietary & Low IDDS & 90 & 46.9 & 95 & 42.4 \\
\hline \multirow[t]{2}{*}{ diversity Score } & Middle IDDS & 45 & 23.5 & 80 & 35.7 \\
\hline & High IDDS & 44 & 29.6 & 49 & 21.9 \\
\hline \multirow[t]{2}{*}{ Loss of weight } & No & 110 & 62.0 & 99 & 44.2 \\
\hline & Yes & 69 & 38.0 & 125 & 55.8 \\
\hline \multirow{2}{*}{$\begin{array}{l}\text { HIV/AIDS co } \\
\text { morbidity }\end{array}$} & No & 169 & 94.4 & 188 & 83.9 \\
\hline & Yes & 10 & 5.6 & 36 & 16.1 \\
\hline \multirow[t]{2}{*}{ Kalazar } & No & 174 & 97.2 & 202 & 90.2 \\
\hline & Yes & 5 & 2.8 & 22 & 9.8 \\
\hline \multirow[t]{2}{*}{ TB } & No & 162 & 90.5 & 189 & 84.4 \\
\hline & Yes & 17 & 9.5 & 35 & 15.6 \\
\hline
\end{tabular}

Divorced and single respondents were 2.7 [AOR 2.768, 95\% CI $(1.338,5.729)]$ and 2.4 [AOR 2.445 , CI 95\% $(1.367,4.373)]$ times more likely to be malnourished respectively as compared to those married.

The patients who have loss of weight for the last three months were 1.6 times more likely to be malnourished when compared to those have no loss of weight for the last three months [1.609, 95\% CI (1.026, 2.524)].

Regarding to patients morbidity status patients who have HIV/ AIDS co-infection 2.3 times more likely to be malnourished when compared to those patients who have no HIV/AIDS co-infection [2.318, 95\% CI (1.024,5.244)] (Tables 2 and 3).

\section{Discussion}

This study showed that the prevalence of malnutrition among adult hospitalized patient was $55.6 \%$. This finding is high when we compare it with a study carried out in Portugal 9.7\% [10] and China (17.8\%) [11]. The difference may be explained by differences in the socioeconomic status, study area sample size of the study and geographical area of study setting [12-15].

In this paper factors found to be significant to malnutrition was literacy level; illiterates which is comparable with a study done in Portugal, in Rwanda and Dilla university referral hospital; illiterate and [16-21]. The possible explanation could be literacy level may be predictor of awareness of dietary diversity, additionally educated people are more likely to access balanced diet.

In this study weight loss was found to be significantly associated with malnutrition. Which is low a study done in Netherland [17]. And São Paulo [19]. This might be the result of; unhealthy weight loss may increase the chance of under nutrition and effect of medication on appetite.

In the current study, the other factor found to be significant to malnutrition was marital status, single, divorced which is comparable with a study conducted in Portugal single/divorced/widowed [16]. In the current study context all hospitalized patients are expected to get

Table 3. Results from Logistic Regression analysis of selected characteristics and associated malnutrition in adult Hospitalized patients, ANRS Referral Hospitals, Ethiopia, 2014.

\begin{tabular}{|c|c|c|c|c|c|}
\hline \multicolumn{2}{|l|}{ Variables } & Normal & Malnutrition & COR(95\%CI) & AOR $(95 \% C I)$ \\
\hline \multirow[t]{3}{*}{ Marital Status } & Married & $127(75.6)$ & $128(57.1)$ & 1 & 1 \\
\hline & Single & $28(16.7)$ & $58(25.9)$ & $2.055(1.230,3.435)^{*}$ & $2.445(1.367,4.373)^{* *}$ \\
\hline & Divorced & $13(7.7)$ & $38(17.0)$ & $2.900(1.475,5.701)^{*}$ & $2.768(1.338,5.729)^{* *}$ \\
\hline \multirow[t]{3}{*}{ Age } & $18-40$ & $70(39.1)$ & $111(49.6)$ & $0.679(0.442,1.041)^{*}$ & \\
\hline & 41-64 & $79(44.1)$ & $85(37.9)$ & $0.589(0.324,1.068)^{*}$ & \\
\hline & $>64$ & $30(16.8)$ & $28(12.5)$ & 1 & \\
\hline \multirow[t]{2}{*}{ Family Size } & $<4$ & $49(27.4)$ & $91(40.6)$ & 1 & \\
\hline & $\geq 4$ & $130(72.6)$ & $133(59.4)$ & $0.551(0.361,0.841)^{*}$ & \\
\hline \multirow[t]{2}{*}{ Education } & Illiterate & $100(59.5)$ & $160(71.4)$ & $1.700(1.114,2.595)^{*}$ & $2.046(1.261,3.321)^{* *}$ \\
\hline & Literate & $68(40.5)$ & $64(28.6)$ & 1 & 1 \\
\hline Individual & -LDDS & $79(47.0)$ & $100(44.6)$ & $1.438(0.871,2.374)^{*}$ & $3.690(1.946,6.995)^{* *}$ \\
\hline Dietary & -MDDS & $39(23.2)$ & $80(35.7)$ & $2.331(1.335,4.070)^{*}$ & $1.816(1.024,3.220)^{* *}$ \\
\hline Diversity Score & -HDDS & $50(29.8)$ & 44 (19.6) & 1 & 1 \\
\hline \multirow[t]{2}{*}{ Loss of weight for the last 3 months } & No & $105(62.5)$ & $99(44.2)$ & 1 & 1 \\
\hline & Yes & $63(37.5)$ & $125(55.8)$ & $2.104(1.398,3.167)^{*}$ & $1.609(1.026,2.524)^{* *}$ \\
\hline \multirow[t]{2}{*}{ HIV/AIDS co-morbidity individual } & No & $159(94.6)$ & $187(83.5)$ & 1 & 1 \\
\hline & Yes & $9(5.4)$ & $37(16.5)$ & $3.496(1.637,7.463)^{*}$ & $2.318(1.024,5.244)^{* *}$ \\
\hline \multirow[t]{2}{*}{ Kalazar } & No & $174(97.2)$ & $202(90.2)$ & 1 & \\
\hline & Yes & $5(2.8)$ & $22(9.8)$ & $3.790(1.406,10.220)^{*}$ & \\
\hline \multirow[t]{2}{*}{ TB } & No & $162(90.5)$ & $189(84.4)$ & 1 & \\
\hline & Yes & $17(9.5)$ & $35(15.6)$ & $1.765(0.953,3.268)^{*}$ & \\
\hline
\end{tabular}


care and dietary support from both the hospital and the family; while single and divorced were less likely to get care from their family due to different reason, so that they may be affected psychosocially, feeding habit and full filling daily requirements.

The current study identifies that the presence of HIV co/morbidity was a risk factor for malnutrition; it was also identified as risk factor in study carried out in Dilla University Referral Hospital [21]. The difference might be due to chronic conditions, severity of illness or stage of HIV disease progression.

In this study Dietary diversity score is found to be significant and associated with malnutrition, Hospitalized patients with Low DDS, Medium DDS. This finding could be explained by the fact that inadequate and imbalance essential nutrients diet intake is a predictor for under nutrition [22-27].

\section{Conclusion}

Prevalence of malnutrition in adult hospitalized patient was found to be high and marital status, education, weight loss for the last three months, Individual Dietary Diversity Score, and HIV co-morbidity were found to be associated with under nutrition.

\section{Authors' contributions}

AH wrote the proposal, participated in data collection, analyzed the data and drafted the paper. MH and ET approved the proposal with some revisions, participated in data analysis and revised subsequent drafts of the paper. All authors read and approved the final manuscript.

\section{Acknowledgements}

We are very grateful to University of Gondar for financial support and all admitted patients who participated in this study for their commitment in responding to our interviews.

\section{References}

1. Borton DC (2013) Malnutrition /Doctor/patient Co.UK. UK Clinical Guidelines.

2. Lisa A. Barker, Timothy C Crowe (2011) Hospital Malnutrition:prevalence,identificati on \& impact on patients and the health care system. Internal Journal of Environmental Research and Public Health.

3. Aeberhard C, Stanga Z, Leuenberger M (2014) Practical scores for the detection of malnutrition. Ther Umsch 71: 141-147. [Crossref]

4. Álvarez-Hernández J, Planas Vila M, León-Sanz M, García de Lorenzo A, CelayaPérez S, et al. (2012) Prevalence and costs of malnutrition in hospitalized patients; the PREDyCES Study. Nutr Hosp 27: 1049-1059. [Crossref]

5. Fessies AT (2008) Malnutrition: Aserious concern for Hospitalize patients. Today's Dietitian, 10: 44.

6. SL Lim, YH Chan, WC Loke, M Ferguson, L Daniels. Malnutrition and its impact on cost of hospitalization, length of stay, readmission and 3 years mortality.

7. Aquino Rde C, Philippi ST (2011) Identification of malnutrition risk factors in hospitalized patients. Rev Assoc Med Bras 57: 637-643. [Crossref]

8. Álvarez-Hernández J, Planas Vila M, León-Sanz M, García de Lorenzo A, CelayaPérez S, et al. (2012) Prevalence and costs of malnutrition in hospitalized patients; the PREDyCES Study. Nutr Hosp 27: 1049-1059. [Crossref]

9. Michelle A Mendez CAM, Barry M Popkin (2005) Overweight exceeds underweight among women in most developing countries 1-3. The American Journal of Clinical Nutrition 81: 21. [Crossref]

10. Amaral TF, Matos LC, Teixeira MA, Tavares MM, Alvares L, et al. (2010) Undernutrition and associated factors among hospitalized patients. Clin Nutr 29: 580585. [Crossref]

11. Fang S, Long J, Tan R, Mai H, Lu W, et al. (2013) A multicentre assessment of malnutrition, nutritional risk, and application of nutritional support among hospitalized patients in Guangzhou hospitals. Asia Pac J Clin Nutr 22: 54-59. [Crossref]

12. Xiaokun Liang, Marie T Nolan, Xinjuan Wu MSN, Haiyan Zhang MSN, Yining Zheng BSN, et al. Nutritional risk, malnutrition.

13. van Nie-Visser NC (2014) Which characteristics of nursing home residents influence differences in malnutrition prevalence? An international comparison of The Netherlands, Germany and Austria. Br J Nutr 111: 1129-1136. [Crossref]

14. Hadgu TH, Worku W, Tetemke D, Berhe H (2013) Undernutrition among HIV positive women in Humera hospital, Tigray, Ethiopia, 2013: antiretroviral therapy alone is not enough, cross sectional study. BMC Public Health 13: 943. [Crossref]

15. Belaynew W. Taye (2012) Adult Nutritional Status and Factors Associated with Malnutrition Amon PLHIV in UoG Referral Hospital 13th world Congress on public Health.

16. Faculdade de Ciências da Nutrição e Alimentação da Universidade do Porto, R.D.R.F., 4200-465 Porto, Portugal. amaral.tf@gmail.com, Undernutrition and associated factors among hospitalized patients. Clin Nutr. , 2010. 10(05): 580-585.

17. Loh KW, Vriens MR, Gerritsen A, Borel Rinkes IH, van Hillegersberg R, et al. (2012) Unintentional weight loss is the most important indicator of malnutrition among surgical cancer patients. Neth J Med 70: 365-369. [Crossref]

18. Gyung-Ah Wie, Yeong-Ah Cho, So-Young Kim, Soo-Min Kim, Jae-Moon Bae, et al. (2010) Prevalence and risk factors of malnutrition among cancer patients according to tumor location and stage in the National Cancer Center in Korea. Applied nutritional investigation 26: 263-268. [Crossref]

19. Aquino Rde C, Philippi ST (2011) Identification of malnutrition risk factors in hospitalized patients. Rev Assoc Med Bras 57: 637-643. [Crossref]

20. Nicole Sirotin, DH, Segal-Isaacson CJ, Qiuhu Shi, Adebola Adedimeji, Eugene Mutimura, et al. (2012) Structural determinants of food insufficiency, low dietary diversity and BMI: a cross-sectional study of HIV-infected and HIV-negative Rwandan women BMJ open.

21. Solomon Hailemariam (2013) Malnutrition: prevalence and its associated factors in people living with HIV/AIDS, in Dilla Univesity Referal Hospital. Archives of Public Health 71.

22. Mengesha B, Endris M, Takele Y, Mekonnen K, Tadesse T, et al. (2014) Prevalence of malnutrition and associated risk factors among adult visceral leishmaniasis patients in Northwest Ethiopia: a cross sectional study. BMC Res Notes 7: 75. [Crossref]

23. Kruizenga HM, Seidell JC, de Vet HC, Wierdsma NJ, van Bokhorst-de van der Schueren MA (2005) Development and validation of a hospital screening tool for malnutrition: the short nutritional assessment questionnaire (SNAQ). Clin Nutr 24: 75-82. [Crossref]

24. Tappenden KA, Quatrara B, Parkhurst ML, Malone AM, Fanjiang G, et al. (2013) Critical role of nutrition in improving quality of care: an interdisciplinary call to action to address adult hospital malnutrition. JPEN J Parenter Enteral Nutr 37: 482-497. [Crossref]

25. Swindale A, Bilinsky P (2005) in HDDS (Household Dietary Diversity Score) for measurement of household food access: Indicator Guide, Food and Nutrition Technical Assistance (FANTA) Project: Washington, D.C.

26. Steendij JSR (2012) The International Wealth Index (IWI), in Nijmegen Center for Economics (NiCE) Institute for Management Research Radboud University Nijmegen: Netherlands.

27. Gina Kennedy (2011) Guidelines for Measuring Household and Individual Dietary Diversity, FAO, European Union, through the EC-FAO.

Copyright: (C2015 Haile A. This is an open-access article distributed under the terms of the Creative Commons Attribution License, which permits unrestricted use, distribution, and reproduction in any medium, provided the original author and source are credited. 